\title{
A Contemporary Proportional Exploration of Numerous Routing Protocol in VANET
}

\author{
Yatendra Mohan Sharma \\ Research Scholar, Department of Computer Science, \\ Banasthali University, Jaipur (Rajasthan), India,
}

\author{
Saurabh Mukherjee \\ Phd,Associate Prof. Department of Computer \\ Science, Banasthali University, Jaipur, (Rajasthan), \\ India,
}



Fig. 1 : Representation of a Vehicular Adhoc Network [3]

This paper presents the current exhaustive investigation of numerous routing protocols and ongoing research in VANET with their merits and shortcomings, which can be used for further enhancement or development of efficient routing protocol. The paper is organized as, section 2 present the numerous routing protocol in VANET. Section 3 describes the recent proposed work of researchers. Current issues in VANET routing protocol is represent in section 4 and finally paper is concluded in section 5 .

\section{ROUTING PROTOCOL IN VANET}

The highly dynamic characteristic of VANET makes challenge in designing process of efficient routing protocols. Various hoc routing protocols have been proposed in recent years. These protocols enable the exchange of data between distinct pairs of nodes, using intermediate network participants for forwarding packets on their way to the destination. In VANET, the routing protocols are classified into five categories: Topology based routing protocol, Position based routing protocol, Cluster based routing protocol, Geocast routing protocol and Broadcast routing protocol. These protocols are characterized on the basis of area / application where they are most suitable [4]. A broad view of VANET routing protocol can be seen in figure 2 .

Fig. 2 : Routing Protocol in VANET


\begin{abstract}
MANET not suitable therefore researchers concentrate on creation of novel approaches that can be implemented and effectively work in VANET environment. A VANET overview can be seen in figure 1.

1. INTRODUCTION

emerging, particularly challenging class of Mobile Ad Hoc Networks (MANETs). In VANET technology of Wi-Fi, evolving standard for communication in between nodes like vehicle to vehicle and vehicle to road side unit.

The wheel network make the architecture of VANET in which can be used internet facility by using road side units. Some unique characteristics of VANET like high mobility, road topology, no boundaries of network size make it different from other ad-hoc network like MANET and make challenging environment to establish reliable networks. The topology and other research work that is done on MANET cannot be directly applied in VANET network due to fundamental difference in between VANET \& MANET. Hence new models is requires in VANET for the communication behavior investigation [1] [2]. The wireless local area network, pure ad-hoc network and hybrid categories comprise the architecture of VANET. Due to
highly dynamic nature of VANET existing routing protocol of
\end{abstract}




\subsection{Topology Based Routing Protocols}

Topology-based routing protocols rely on the topology of the network. The most of topology based protocol tries to used minimum level computing time for forward a packet in network and make balance between potential routes. These routing protocols use link's information and store that information in table before sending data from source to destination node. They are further divided into two categories shown in fig. 3.

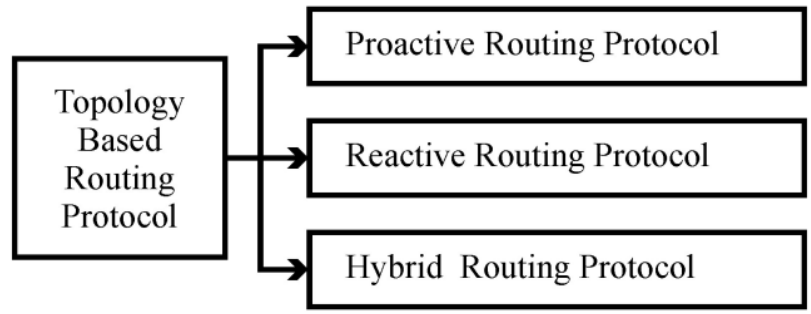

Fig. 3 : Topology Based Routing Protocol in VANET

\subsubsection{Proactive Routing Protocol}

The proactive protocol is also known as table driven routing protocol. For data communication in between all the nodes of network these protocols use periodic exchanged knowledge of topology. For this these protocol use huge amount of bandwidth to keep information about periodic updates of topology but do not have delay in initial route discovery [4]. These protocol maintained reliable and up to date information at each node in network [5]. There are several routing protocols that fall under this category shown in fig. 4.

\section{Table Driven Protocol}

$\longrightarrow$ Destination Sequence Distance Vector (DSDV)

$\rightarrow$ Optimized Link State Routing (OLSR)

$\rightarrow$ Fisheye State Routing (FSR)

$\rightarrow$ Global State Routing (GSR)

$\rightarrow$ Source Tree Adaptive Routing (STAR)

$\rightarrow$ Wireless Routing Protocol (WRP)

Fig. 4 : Proactive Routing Protocol in VANET

DSDV: It is a table-driven routing protocol for ad-hoc mobile networks based on the Bellman- Ford algorithm. [6]. In this route is maintained through periodic and event triggered routing table exchanges. Each node maintains a next-hop table, and neighbors exchange it in network for update information. It eliminates route looping, increases convergence speed and used setting time to reduce control message overhead. It performs best in low mobility environment because of each node in network need to maintain a complete list of routes that produce higher overhead of network.

OLSR: It is an optimization of a pure link state protocol for mobile ad hoc networks. The protocol inherits the constancy of a link state algorithm and provide route immediately when needed. OLSR does not require reliable transmission and sequenced delivery of control messages, each node in network sends control data periodically without transmission reliability therefore sustain a reasonable loss of some such messages. OLSR protocol minimizes the overhead by selects a set of neighbor nodes called as multipoint relays (MPR) which retransmits its packets. The neighbor nodes which are not in its MPR set can only read and process the packet. This procedure reduces the number of retransmissions in a broadcast procedure [7].

FSR: The functionally of FSR is similar to Link State Routing protocol in that this protocol maintain a routing table as maintaining in Link State Routing protocol with the difference of the way in which routing information in disseminated. In LS, link state packets are generated and flooded into the network whenever a node detects a topology change. In FSR, link state packets are not flooded and nodes exchange packet proactively, not at the time of receiving route request. [8].

GSR: Like FSR protocol the GSR is also link state based routing protocol. It has the similar functionality as DSDV and avoiding the flooding of link state packets. In GSR every node has a updated routing topology table that it exchange with local neighbor node only. At the time of receiving routing message node update its topology table after checking the packet sequence number. If number is not found in table than node update its routing table but if number is found in table than discard that packet. After successfully updating routing table node broadcasts the information to its neighbors.

STAR: It is a table-driven routing protocol in which every node maintains network topology information and builds shortest path tree to store the preferred shortest path from source to destination. The basic mechanisms in STAR include the detection of neighbors and exchange of topology information (update message) among nodes [9].

WRP: In this routing protocol every node contain the in formation of routing table, link cost table, distance table and message retransmission list table for keeping routing information among all the nodes of network [10].

\subsubsection{Reactive Routing Protocol}

This category of routing protocols is also called on-demand routing protocols. It periodically updates the routing table, when some data is there to send. But these protocols use flooding process for route discovery, which causes more routing overhead and also suffer from the initial route discovery process, which make them unsuitable for safety applications in VANET. There are several routing protocols that fall under this category shown in fig. 5 .

\section{On-Demand Protocol}

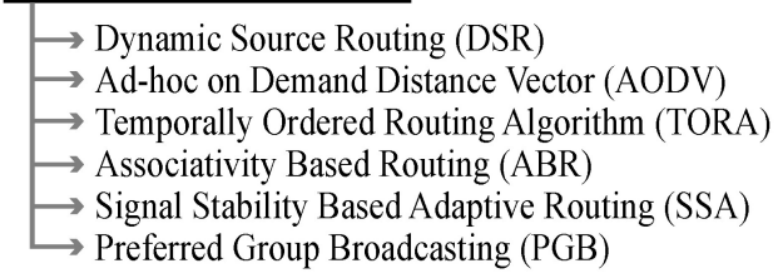

Fig. 5 : Reactive/On-Demand Routing Protocol

DSR: Dynamic Source Routing (DSR) is a routing protocol based on a method known as source routing and designed specifically for multi-hop wireless ad-hoc network. Unlike other ad-hoc network protocol DSR not require any periodic routing message in network. In network for sending message this protocol store the route from source to destination node in a route cache and not depend at the information of mediator node routing table. In this protocol path length effect the routing overhead and broken links in network does not repair locally at route maintenance process. This is the main limitation of this protocol that makes it unsuitable for large high mobility network. It has two important phases, route discovery and route maintenance $[11,12]$.

AODV: This protocol combines the some functionality of DSR and DSDV protocol and establishes a route at only on-demand, when a node requires sending data packets. For maintaining routing information the AODV use routing tables but it has not maintain routes information for all of nodes that are not communicating in network. This protocol can be used for 
unicast \& multicast routing. AODV handles route discovery process with Route Request (RREQ) messages [13].

TORA: Temporally Ordered Routing Algorithm (TORA) belong belongs to a family of link reversal routing algorithms where source node act as root of the tree and create a directed acyclic graph towards the destination that directs the flow of packets and ensures their reachability to all the nodes. This protocol controls the message propogation in highly dynamic environment and performs three tasks creating of path, path maintenance and erasure of the route when the route is no longer valid. If a link goes fail TORA propagates control message only around the point of failure. While other ad-hoc network protocols re-initiate the route discovery. A node constructs the directed graph by broadcasting a query packet. Upon receiving a query packet it will broadcast a reply packet, if it has a downward link to the destination; otherwise, it simply drops the packet [14].

ABR: ABR defines a new metric for routing known as the degree of association stability. The association stability represents stability of connection in between two nodes in network. To express its associativity each node broadcast periodically a beacon to indicate its presence and at the time of receiving of beacon the node counter is updated. A low state of node mobility can be indicates with a high value of counter and a high state node mobility may indicates with a low value of associativity counter. The counter of associativity is reset when the neighbors of a node or the node itself move out of proximity. The fundamental objective of ABR is to find longerlived routes for ad hoc mobile networks. The three phases of ABR are Route discovery, Route reconstruction (RRC) and Route deletion [15].

SSA: It is an on demand routing protocol that rectifying the link stability by using its signal strength and show that link is stable or unstable. In comparison of other ondemand routing protocol like AODv and DSR that provides shortest path in network whenever needed the SSA protocol provides more stable route. This protocol provides good link stability as compared to other protocols [16].

PGB: This protocol is broadcasting mechanisms that reduce broadcast overhead that is associated with AODV route discovery mechanisms. PGB provide route stability especially for VANETs environments. On the basis of received signal receivers can determine which one in the group to broadcast and whether they are in the preferred group [17].

\subsubsection{Hybrid Routing Protocols}

The hybrid protocols are introduced to reduce the control overhead of proactive routing protocols and decrease the initial route discovery delay in reactive routing protocols. The routing protocols that fall under this category shown in fig. 6

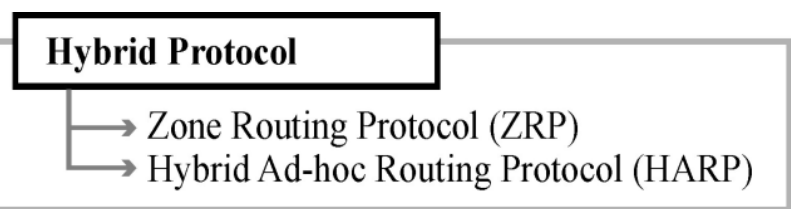

Fig. 6 : Hybrid Routing Protocol

ZRP: This protocol divides the whole network into the zone. In a network zone is a group of nodes which are in a radius. Zone radius size depend on its length $\alpha$ where $\alpha$ is the number of hops to the perimeter of the zone. In ZRP, a proactive routing protocol (IARP) is used in intra-zone communication and an inner-zone reactive routing protocol (IARP) is used in intrazone communication. Source sends data directly to the destination if both are in same routing zone otherwise IERP reactively initiates a route discovery. ZRP aims to find loop free routes to the destination. It uses border casting method to construct multicast trees to flood the query packets instead of standard flooding to discover the destination route [18].

HARP: Like ZRP this protocol also divides the network in zone with the key difference that ZRP divide network in the overlapping zones while this protocol divides entire network into non-overlapping zones. This protocol has aim to improve delay in between the source and destination node by providing the stable route. It applies route discovery between zones to limit flooding in the network, and choose best route based on the stability criteria. [19].

\subsection{Position Based Routing Protocols}

The position based protocols use of nodes location information instead of links information to routing. Position based routing consists of class of routing algorithm. They share the property of using geographic positioning information in order to select the next forwarding hops. The packet is send without any map knowledge to the one hop neighbor, which is closest to destination. Position based routing is beneficial since no global route from source node to destination node need to be created and maintained [20]. It broadly divided in two types shown in fig. 7

\begin{tabular}{|l|l|}
\hline $\begin{array}{c}\text { Position } \\
\text { Based } \\
\text { Routing } \\
\text { Protocol }\end{array}$ & Position Based Greedy V2V Protocols \\
\hline Delay Tolerant Protocols \\
\hline
\end{tabular}

Fig. 7: Broad View of Position Based Routing Protocol

\subsubsection{Greedy V2V/NON DTN Protocol}

The routing protocols that fall under this category shown in fig. 8.

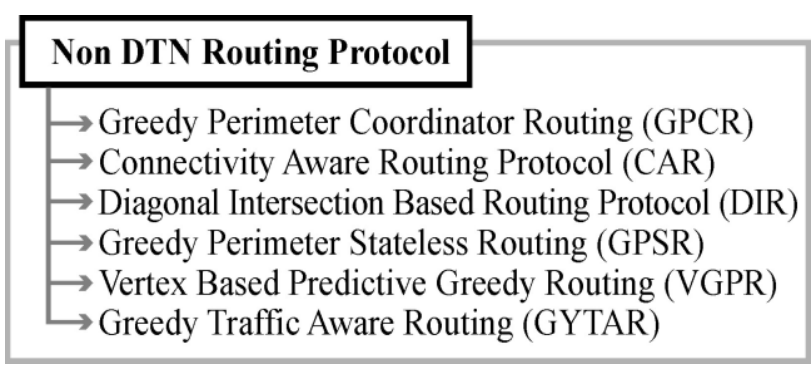

Fig. 8 : Greedy/Non DTN Routing Protocol

GPCR: GPCR protocol is very well suited for highly dynamic environments such as inter-vehicle communication on the highway or city. By using the restricted greedy forwarding procedure the GPCR protocol forward data packet and does not requires any external or global information. This represent that in this protocol when choosing the next hop, a coordinator node (a node on a junction) is preferred to a non-coordinator node, even if it is not the closest node to destination [21].

CAR: This protocol is designed for city and highway environment. To minimize broadcast For broadcast minimization from AODV route discovery and Advanced Greedy Forwarding (AGF) to account for node mobility, Naumov et al. presented Connectivity-Aware Routing (CAR) in VANETs. This protocol uses PGB for data dissemination mode and for path discovery it use AODV protocol with the guard concept for maintaining the path. It has unique characteristics that it maintains the cache of successful route between various source and destination pairs [22]. 
DIR: This protocol improves the functionality of CAR protocol. In between the source and destination mobile node this protocol constructs a series of diagonal intersections. In comparison of CAR this protocol may set fewer anchors and and can automatically adjust routing path for keeping the lower packet delay, compared to CAR protocol [23].

GPSR: It is a routing protocol that takes the packet forwarding decision on the basis of exploiting of position of mobile node and the packet destination location. In GPSR, the positions of neighbors are maintained by the intermediate node without keeping routing metrics, which makes the protocol stateless. GPSR comprises two modes: greedy forwarding mode and perimeter mode [24].

VGPR: This protocol is especially designed for urban environment. The VGPR protocol is a multi-hop that has the functionality of vehicle to infrastructure routing. This protocol forward packets from mobile node to fixed node via sequence of junctions. For estimation of sequence of valid junctions this protocol uses position, velocity and direction parameters. In comparison of other this protocol has less control overhead. Due to fair path selection, lesser packets are to be retransmitted and the reliability and packet delivery ratio are improved [25].

GyTAR: This routing protocol is an improved greedy traffic aware routing strategy in city environment for finding strong routes between source and destination nodes. GyTAR considers vehicle direction, vehicle velocity, multi-directional roads, and the changing traffic environment into its routing strategy. It contains two modules: Junctions selection, forwarding data between two junctions. Each vehicle maintains a neighbor table in which the position, velocity, and direction of each neighbor vehicle are recorded. Thus, when a packet is received, the forwarding vehicle computes the new predicted position of each neighbor using the recorded information (velocity, direction, and the latest known position), and then selects the next hop neighbor, which is the closest to the destination junction. Drawback of this protocol is loop problem and forwarding of nodes in wrong direction [26].

\subsubsection{Delay Tolerant / DTN Protocol}

The routing protocols that fall under this category shown in fig. 9.

\section{DTN Routing Protocol}

Motion Vector Routing Algorithm (MOVE)

Vehicle Assisted Data Delivery (VADD)

Static Node Assisted Adaptive Routing Protocol (SADV)

Fig. 9 : DTN Routing Protocol

MOVE: This algorithm is specially designed for sparse networks and for vehicles that transfer data from sensor networks to base station. The mobile nodes in this environment behave as a router whose having the intermittent connectivity from the other node in network. This protocol takes an assumption that every mobile node contains the information of its own position, heading and destination. From this information the current vehicle node can calculate the closest distance between the vehicle and message destination [27].

VADD: This protocol uses the predictable mobility of node and work at the carry and forward approach. Among proposed VAAD protocols H-VAAD shows better performance. In this protocol a mobile node makes decision of packet forwarding at a junction and selects the next forwarding path with the smallest packet delivery delay. A path is simply a branched road from an intersection [28].

SADV: This protocol is designed for sparse network to reduce the packet delivery delay and like other this protocol also adapts varying traffic density dynamically by allowing each node to measure the amount of time for message delivery. Like MOVE this protocol also has the assumption that each mobile node in network knows its position through GPS and each node has accessed to external static street map. SADV has three different modules; Static Node Assisted Routing (SNAR), Link Delay Update (LDU) and Multipath Data Dissemination (MPDD). The two modes operate in SADV: "In Road Mode" and "Intersection Mode". SNAR make use of optimal paths, which are determined on the basis of graph abstracted from road map. LDU maintains the delay matrix dynamically by measuring the delay of message delivery between static nodes. MPDD helps in multipath routing [27].

\subsection{Cluster Based Routing Protocols}

In cluster based routing protocols the formation of clusters and the selection of the cluster-head is an important issue. In this protocol, the geographic area is divided into some foursquare grids. Only if there is a vehicle in a grid will a vehicle be elected to the cluster header, and the data packet is routed by cluster header across some grids one by one. The various cluster based routing protocol are shown in fig. 10.

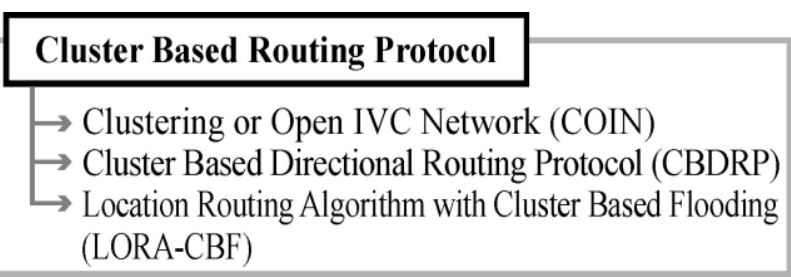

Fig. 10 : Cluster Based Routing Protocol

COIN: Unlike other conventional clustering method where ID is used for selection of cluster head, in COIN protocol it depends at vehicle driver intension and the dynamicity of vehicle node. Inter vehicle communication also accommodates the oscillatory nature of inter-vehicle distances. Ideally, the relative mobility between a cluster head and a member node should be low, so they remain in radio contact for as long as possible [29].

CBDRP: This protocol make cluster by dividing the network node whose moves in the same direction in the network and for forwarding the information the packet is send to head of cluster and then that packet is forward to the header which is in the same cluster with the destination. Like CBR protocol the working of CBDRP is same for selection of the head in cluster and route maintaining but it considers velocity and direction of a vehicle [30].

LORA-CBF: In this protocol designed each node of cluster in network may be a cluster head, member or can perform role of gateway to communicate with other cluster head. Every head of cluster maintains tables that contain the location and address of other members and gateway node of cluster. This is incorporate dynamic movement scheme expected decisions of driver under certain scenario, enhancing the tolerance limit of inter-vehicle distances to provide more stable structure at the cost of little additional overhead. As like method used in greedy routing protocol for packet forwarding, in this protocol also only cluster head and gateways can send out the location request (LREQ) packets [31].

\subsection{Geocast Routing Protocols}

These protocols are used to send a message to all vehicles in a pre-defined geographical region. The philosophy is that the sender node need not deliver the packet to nodes beyond the ZOR. The scheme followed a directed flooding strategy within a defined ZOR so that it can limit the message overhead. 


\section{Geocast Routing Protocol}

$\rightarrow$ Inter Vehicle Geocast (IVG)

$\rightarrow$ Distributed Robust Geocast (DRG)

$\rightarrow$ Direction Based Geocast Routing Protocol for Query Dissemination in VANET (DG-CASTOR)

\section{Fig. 11 : Geocast Routing Protocol}

IVG: The IVG protocol is used to forward danger information like accident to the entire mobile node that are at highway. Each node in this protocol is associated with timer based mechanism for receiving packet and rebroadcasting of that packet once the timer is elapsed. The location of mobile node and direction of driving determine the risk. The mobile node that comes in a risk area takes a form of multicast group. The speed of node and direction of driving with location defined the multicast group. For reducing the fragmentation of network and packet delivery to the members of multicast group the IVG protocol use functionality of periodic broadcasting. The period of rebroadcasting is calculating based on the maximum vehicle speed. Besides, IVG protocol reduces the hops of delivering message by using the deferring time [32].

DRG: It is a completely distributed geocast protocol that has no state information and the control overhead with the resilient functionality in case of frequent topology change in network. The DRG protocol has objective to enable forwarding of messages as fast and reliable fashion that minimizes the network load [33].

DG-CASTOR: This protocol especially designed for VANET infotainment applications. The protocol make a virtual community Rendez-vous group which is based on future locations prediction of the mobile nodes in the network. However, the query is only disseminated between the nodes belonging to the same Rendez-Vous group [34].

\subsection{Broadcast Routing Protocols}

This is most frequently used routing protocol in VANET especially to communicate the safety related messages. Simplest of broadcast method is carried by flooding in which each node rebroadcast the message to other nodes. This ascertains the arrival of message to all targeted destinations but has a higher overhead cost. The various broadcast routing protocols are shown in fig. 12.

\begin{tabular}{|l|}
\hline Broadcast Routing Protocol \\
\hline \\
$\rightarrow$ BROADCOMM \\
$\rightarrow$ Urban Multihop Broadcast Protocol (UMB) \\
$\rightarrow$ Vector Based Tracing Detection (V-TRADE) \\
$\rightarrow$ Distributed Vehicular Broadcast Protocol (DV-CAST) \\
$\rightarrow$ Edge-Aware Epidemic Protocol (EAEP) \\
$\rightarrow$ Secure Ring Broadcasting (SRB)
\end{tabular}

Fig. 12 : Broadcast Routing Protocol

BROADCOMM: This protocol is suit for highway network at which the entire highway divided in virtual cells which move like mobile nodes. The nodes in the network divided in hierarchy manner in this protocol: the first level includes all the nodes in a cell and the cell reflectors represented the second level of hierarchy. Cell reflected behaves for certain interval of time as cluster head and handles the emergency messages coming from same members of the cell or nearby neighbor. This protocol performs similar to flooding base routing protocols for message broadcasting and routing overhead [35].
UMB: This protocol is designed to overcome problem that often arise in the multi-hop broadcast like hidden node problems in the network during message distribution, packet collision and interference. This protocol handles the looping problem with caching mechanisms. In this protocol without any prior routing topology information the sender mobile node tries to select the furthest node in the broadcast direction for forwarding and acknowledging the packet. It gains high success at higher packet loads and mobile nodes densities [36].

V-TRADE: This scheme is a GPS based message broadcasting protocol. It has the functionality as same of unicast routing protocols Zone Routing Protocol (ZRP). V-TRADE classifies the neighbors into different forwarding groups depending upon position and movement information and for forwarding packet to its neighbor in each group it assigns only few border nodes. It improves the bandwidth utilization but some routing overheads are associated with selecting the next forwarding node in every hop. Because of lesser number of nodes assigned for multihopping, it indicated significant in bandwidth utilization [37].

DV-CAST: It is multi-hop broadcast routing protocol in VANETs and indicates three traffic scenarios for a vehicular broadcasting; dense traffic scenario, sparse traffic scenario, and regular traffic scenario. It is suitable for both of dense and sparse traffic scenarios and reduces the broadcasting overhead. This protocol causes high control overhead and delay in end to end data transfer. In DV-CAST, each vehicle monitors the states of neighboring vehicles all the time to make the broadcasting decisions [39].

EAEP: This is high dynamic and reliable VANEt protocol that reduced control packet overhead by removing the exchanging of additional hello packet for message transfer in between several differ vehicle and eases cluster maintenance. For eliminating beacon message each mobile node piggyback to its location to message broadcast. At the time of receiving a new rebroadcast message, EAEP uses number of transmission from front nodes and back nodes in a given period of time to calculate the probability for making decision whether nodes will rebroadcast the message or not [40].

SRB: This protocol is specialized for broadcasting route requests. For getting stable route the SRB protocol minimize number of retransmission messages.

It divided the mobile node into three groups on the basis of their receiving power as Inner nodes (close to sending node), Outer Nodes (far away from sending node), Secure Ring Nodes (preferable distance from sending node). The goal of SRB protocol is not only to minimize broadcasting messages but also to get more stable routes [41].

\section{RECENT PROPOSED APPROACH IN VANET}

In [42] a model is proposed that identifies the most relevant communication aspects and have also identified the major threats. In their work by analyzing their robustness of proposed model they shown that public key cryptography is fit for the security of these networks. In [43] authors proposed a set of design principles for future security and privacy solutions for vehicular communication systems. They show the sketch of security requirements for the vehicular communication systems and provide models for the system and communication, as well as models for the adversaries.

In [44] security architecture is present that follows a clean and modular design. In this paper they identify the stakeholders and their responsibilities and after that they emphasis on the functional layer view and highlight the concepts which jointly secure the vehicular communication. On the basis of these concepts, they present an implementation of proposed approach 
which introduces the security concepts into the protocol stack of vehicular communication system. In [45] authors present security architecture for vehicular communication with the objectives of the architecture include the management of identities and cryptographic keys, the security of communications, and integration of privacy enhancing technologies. Their design approach aims at a system that relies on well-understood components which can be upgraded to provide enhanced security and privacy protection in the future. In [46], protocol "edge node-based directional routing (E-DIR)" is proposed that uses edge nodes with DIR protocols. In this authors examine the significance of position-based routing using edge nodes for forwarding the data in a vehicular ad hoc network. This approach minimizes the path length by minimizing the number of hops between source and destination vehicles. In [47], authors proposed intersection-based routing protocol finds a minimum delay routing path in various vehicle densities. Moreover, vehicles reroute each packet according to real-time road conditions in each intersection, and the packet routing at the intersections is dependent on the moving direction of the next vehicle. The proposed (IBR) protocol has less end-to-end delay compared to vehicle-assisted data delivery (VADD) and greedy traffic aware routing protocol (GyTAR) protcols.

In [48], authors present a model for information dissemination technique for VANET that may enhance the routing performance in terms of key performance indicator. This is managed by calculating most stable link for information dissemination between the communicating vehicles. The decision regarding the stability of link is assisted by adjusting the weights for three key selection parameters (velocity distance and position). Consideration of the higher value of weight function for each vehicle, link stability and life time of sending link will be helpful in improving the performance of this model.

In [49], A novel routing scheme, Anchor-Geography based routing protocol (AGP), designed specifically for VANET communication in city environment is proposed. AGP protocol gains improvement in packet delivery ratio and average hops. In [50] a novel routing protocol is proposes in VANET for sparse environment called Vehicle Second Heading Direction Routing Protocol (VSHDRP), which is designed to leverage the probability of delivering a data packet to its destination and to increase connectivity and route stability by utilizing the knowledge of the Second Heading Direction (SHD) in the process of selecting the next-hop node. This new routing protocol contains two modes; the highway straight mode and the roundaboutlintersection mode. Moreover, the two modes of VSHDRP protocol are formalized in the Calculus of Contextaware Ambients (CCA) and simulated using the CCA interpreter ccaPL in order to analyses and validate the behavior of the protocol.

\section{CURRENT ISSUES IN VANET RTOUTING PROTOCOLS}

In a vehicular ad hoc network it is important that broadcast messages are received with a high probability. Broadcast messages such as emergency warnings should be received by all vehicles in the proximity of the endangered. The existing routing protocols are effective only when the node population is small.

The Reactive routing schemes will fail to discover a complete path due to frequent network partition. These protocols periodically update the routing table, when some data is there to send. But these protocols use flooding process for route discovery, which causes more routing overhead and also suffer from the initial route discovery process, which make them unsuitable for safety applications in VANET.
The proactive routing protocols have advantage that No Route Discovery is required and low latency for real time applications but shortcoming of this protocol is that in this protocol unused paths occupy a significant part of the available bandwidth.

The proactive routing protocols will be overwhelmed by the rapid topology changes and even fail to converge during the routing information exchange stage. The Position-based routing schemes generally require additional node physical position information during the routing decision process. A location service is needed as well to provide the position information of nodes.

Due to the high node mobility and the movement constraints of mobile nodes the conventional topology-based routing schemes are not suitable for VANETs.

\section{CONCLUSION}

Routing is an important component in VANET. Due to the high mobility nature the designing an efficient routing protocol for all VANET applications is very difficult. Hence a survey of different VANET protocols is absolutely essential to come up with new proposals for VANET. This paper investigates the numerous routing protocol of VANET with their advantages and shortcomings. Recent proposed models in the area of VANET also present in this paper with the issues that affect the performance of routing protocol to come up new efficient and more reliable protocols for most of the applications in VANET.

\section{REFERENCES}

[1] H.Hartenstein B. Bochow, and D.Vollmer,'Position aware ad hoc wireless networks for inter-vehicle communication the Fleetnet project" i n proc $2^{\text {nd }}$ ACM Int Symp, Mobile Ad hoc Network page : 250-262

[2] Shi, W. Liu Y "Real-Time urba n traffic monitoring with global positioning System equipped vehicles" Intelligent Transport System, IET, Iss ue Date: June 2010, Volume 4 Issue 2 Page :113-120

[3] http://abhi-carmaniacs.blogspot.in/2012/02/vehicular-adhoc-network.html

[4] Jagadeesh Kakarla, S Siva Sathya, B Govinda Laxmi2, Ramesh Babu B3 "A Survey on Routing Protocols and its Issues in VANET" International Journal of Computer Applications (0975 - 8887)Volume 28- No.4, August 2011

[5] S.Tamilarasan, Dr.R.Sivaram "An Analysis and Comparison of Multi-Hop Ad-Hoc wireless Routing Protocols for Mobile Node" International Journal of Science and Applied Information Technology, Volume 1, No.1, March - April 2012

[6] C.E. Perkins, P. Bhagwat, "Highly DSDV", 1994

[7] T. Clausen, et al., "Optimized Link State Routing Protocol (OLSR)”, RFC 3626, Network Working Group, Oct. 2003.

[8] M. Gerla, X. Hong, G. Pei, "Fisheye State Routing Protocol (FSR)", IETF Internet Draft, work in progress, draftietfmanet- fsr-03.txt, July 2002.

[9] J. J. Garcia-Luna-Aceves and M. Spohn, "Source-Tree Routing in Wireless Networks," Proceedings of 7th International Conference on Network Protocols, 1999.

[10] Laiq Khan, Nohman Ayub and Aamir Saeed " Anycast Based Routing in Vehicular Adhoc Networks (VANETS) using Vanetmobisim" World Applied Sciences Journal 7 (11): 1341-1352, 2009 ISSN 1818-4952 (C) IDOSI 
Publications, 2009

[11] D. Johnson, B.D.A. Maltz, and Y.C.Hu, "The Dynamic Source Routing Protocol for Mobile Ad Hoc Networks (DSR)", draft-ietf-manet-dsr-10.txt, 2004.

[12] C.E.Perkins and E. M. Royer. Ad-Hoc On Demand Distance Vector Routing, Proceedings of the 2nd IEEE Workshop on Mobile Computing Systems and Applications (WMCSA), pp. 90-100, 1999.

[13] Zhan Haawei and Zhou Yun.Comparison and analysis AODV and OLSR Routing Protocols in Ad Hoc Network, 2008, IEEE.

[14] Prabhakar Ranjan , Kamal Kant Ahirwar "Comparative Study of VANET and MANET Routing Protocols" Proc. of the International Conference on Advanced Computing and Communication Technologies (ACCT 2011) Copyright (C) 2011 RG Education Society ISBN: 978-981-08-7932-7

[15] http://www.cse.wustl.edu/ jain/cis788-99/ftp/ adhoc_ routing/\#intro

[16] C.E.Perkins, “Ad hoc Networking”, Addison Wesley, 2001.

[17] NAUMOV V, An evaluation of inter-vehicle ad hoc networks based on realistic vehicular traces. MOBIHOC 2006

[18] Z. J. Haas, "The Zone Routing Protocol (ZRP) for ad hoc networks", Internet Draft, Nov. 1997.

[19] Navid Nikaein, Christian Bonnet and Neda Nikaein,"HARP - Hybrid Ad Hoc Routing Protocol", in proceeding of IST 2001: International Symposium on Telecommunications, Iran/Tehran 2001.

[20] Rakesh Kumar, Mayank Dave, "A Comparative Study of Various Routing Protocols in VANET", IJCSI International Journal of Computer Science Issues, Vol. 8, Issue 4, No 1, July 2011 ISSN (Online): 1694-0814

[21] C. Lochert, M. Mauve, H. Fera, and H. Hartenstein, "Geographic routing in city scenarios," ACMSIGMOBILE Mobile Computing and Communications, Vol. 9, 2005, pp. 69-72.

[22] Naumov, V., Gross, T.R. (2007), "Connectivity-Aware Routing (CAR) in Vehicular Ad-hoc Networks," INFOCOM 2007, 26th IEEE International Conference on Computer Communications. IEEE, vol., no., pp.1919- 1927, 6-12 May, 2007.

[23] Y. S. Chen, Y. W. Lin, and C. Y. Pan "A diagonalintersection-based routing protocol for urban vehicular ad hoc networks", Telecommunication System, Vol. 46, 2010.

[24] B. Karp. Gpsr: Greedy perimeter stateless routing for wireless networks. In proceedings of the 6th annual international conference on Mobilecom- puting and networking, pages $243\{254,2000$.

[25] Raj K. Shrestha, Sangman Moh, Ilyong Chung, and Dongmin Choi, "Vertex-based multi-hop vehicle-to infrastructure routing for vehicular ad hoc networks", IEEE proceedings of 43rd Hawaii International Conference on System Sciences (HICSS) 2010.

[26] Moez Jerbi, Rabah Meraihi, Sidi-Mohammed Senouci, Yacine Ghamri-Doudane ENSIIE," GyTAR: improved Greedy Traffic Aware Routing Protocol for Vehicular Ad Hoc Networks in City Environments", VANET'06,
September 2006.

[27] Rakesh Kumar, Mayank Dave " A Comparative Study of Various Routing Protocols in VANET" IJCSI International Journal of Computer Science Issues, Vol. 8, Issue 4, No 1, July 2011 ISSN (Online): 1694-0814

[28] Zhao, J.; Cao, G. (2006), "VADD: Vehicle-Assisted Data Delivery in Vehicular Ad Hoc Networks," INFOCOM 2006. 25th IEEE International Conference on Computer Communications. Proceedings , vol., no., pp.1- 12, April 2006.

[29] Uma Nagaraj, Dr. M. U. Kharat, Poonam Dhamal "Study of Various Routing Protocols in VANET" IJCST Vol. 2, Iss ue 4, Oct . - Dec. 2011

[30] Tao Song, Wei Xia, Tiecheng Song, Lianfeng Shen,"A Cluster-Based Directional Routing Protocol in VANET", International Conference on Communication and Mobile Computing, 2010.

[31] R. A. Santos, "Performance evaluation of routing protocols in vehicular ad hoc networks," 2005.

[32] A. Bachir and A. Benslimane, "A multicast protocol in ad hoc networks inter-vehicle geocast," in Proceedings of IEEE Semiannual Vehicular Technology Conference, Vol. 4, 2003, pp. 2456-2460.

[33] Maria Kihl, Mihail L. Sichitiu, and Harshvardhan P. Joshi "Design and Evaluation of two Geocast protocols for Vehicular Ad-hoc Networks" September 15, 2007; revised February 28, 2008.

[34] Talar Atechian,” DG-CastoR: Direction-based GeoCast Routing Protocol for query dissemination in VANET".

[35] Sandhaya Kohli, Bandanjot Kaur, Sabina Bindra" A comparative study of Routing Protocols in VANET", 2011

[36] G. Korkmaz, "Urban multihop broadcast protocol for intervehicle communication systems"

[37] http://www.personal.psu.edu/akb5073/VANET_note2.pdf

[38] YUN-WEI LIN, YUH-SHYAN CHEN AND SING-LING LEE "Routing Protocols in Vehicular Ad Hoc Networks: A Survey and Future Perspectives", JOURNAL OF INFORMATION SCIENCE AND ENGINEERING 26, 913-932 (2010)

[39] O. Tonguz, N. Wisitpongphan, F. Bai, P. Mudalige, and V. Sadekar, "Broadcasting in VANET," in Proceedings of IEEE Mobile Networking for Vehicular Environments, 2007, pp. 7-12.

[40] M. Nekovee, "Reliable and efficient information dissemination in intermittently connected vehicular ad hoc networks", 2007.

[41] Rainer Baumann, "Vehicular Ad hoc Networks", Master's Thesis in Computer Science, ETH Zurich 2004.

[42] M.C. Schut et al," SOTRIP: A self organizing protocol for traffic information", IWCMC,pp. 21-24, 2009.

[43] Ali Ghazi, Tarik Ozkul," Design and simulation of an artificially intelligent vanet for solving traffic congestion". Masaum journal of basic and applied sciences vol.1, no. 2 September 2009.

[44] Arunkumar Thangavelu et al," A simulated modeling approach towards providing adaptive qos for vehicular 
safety services over vanet", international journal of research and reviews in computer science (IJRRCS), vol. 1, no. 4, december 2010.

[45] Sooksan Panichpapiboon," the future of traffic information systems", journal of information science 70 and technology, vol 1, issue 2, jul-dec 2010.

[46] Ram Shringar Raw, D. K. Lobiyal "E-DIR: a directional routing protocol for VANETs in a city traffic environment" International Journal of Information and Communication Technology archive Volume 3 Issue 3, August 2011. Pages 242-257

[47] Li-Der Chou, Jyun-Yan Yang, Ying-Cheng Hsieh, DerChyn Chang and Chi-Feng Tung "Intersection-Based Routing Protocol for VANETs" Wireless Personal Communications Volume 60, Number 1 (2011), 105-124,

\section{DOI: $10.1007 / \mathrm{s} 11277-011-0257-\mathrm{Z}$}

[48] Masood-ur-Rehman , Halabi Bin Hasbullah and Irshad Ahmad Somro "Enhanced Adjustable Parameter Based Information Dissemination Model (eAPIM) for VANET" Computer Science Journal Volume 1, Issue 1, April 2011

[49] Yan SHI, Xiao-ye JIN, Shan-zhi CHEN “AGP: an anchorgeography based routing protocol with mobility prediction for VANET in city scenarios" The Journal of China Universities of Posts and Telecommunications, Volume 18, Supplement 1, September 2011, Pages 112-117

[50] Moath Muayad Al-Doori, Francois Siewe, and Ali Hilal AlBayatti "Routing Management for DTN Networks in VANET" International Journal of Machine Learning and Computing, Vol. 1, No. 5, December 2011 